Original Research Article

\title{
Comparative effectiveness of sitagliptin and vildagliptin in the management of patients with type 2 diabetes mellitus undergoing haemodialysis: an Indian rural tertiary care centre experience
}

\author{
Suthakaran C. ${ }^{1}$, Bhaskaran S. ${ }^{2 *}$
}

${ }^{1}$ Department of Pharmacology,

${ }^{2}$ Department of Medicine, Melmaruvathur Adhi Parasakthi Institute of Medical Sciences \& Research, (Affiliated to The Tamilnadu Dr MGR Medical University), Melmaruvathur 603319, Kancheepuram, Tamilnadu, India

Received: 28 May 2018 Accepted: 09 June 2018

*Correspondence to:

Dr. Bhaskaran S.,

Email: bhprem@hotmail.com

Copyright: () the author(s), publisher and licensee Medip Academy. This is an openaccess article distributed under the terms of the Creative Commons Attribution NonCommercial License, which permits unrestricted noncommercial use, distribution, and reproduction in any medium, provided the original work is properly cited.

\begin{abstract}
Background: The prevalence of chronic kidney disease is increasing with diabetic nephropathy as the common underlying cause. Although numerous drugs are being used to improve glycaemic control, evidence in patients with diabetic nephropathy is sparse. The aim of the present was to evaluate the effectiveness of sitagliptin or vildagliptin addition on glycaemic control in patients with T2DM undergoing haemodialysis as part of their routine care in a rural tertiary care setting.

Methods: Type 2 diabetic patients on maintenance haemodialysis as part of routine care and whose glycaemia was not controlled adequately and prescribed one of the oral gliptins once daily in addition to existing therapy for a period of 24 weeks were included in the present study. Effectiveness was assessed in terms of glycaemic control as measured by the change over time in glycated haemoglobin. Data analysis included glycated haemoglobin, body weight, serum creatinine, urine albumin creatinine ratio and the occurrence of hypoglycaemia.

Results: Significant reduction in glycated haemoglobin values were noted after 24 weeks of therapy with gliptins similar to insulin glargine with a small weight loss. There was an insignificant decrease in the serum creatinine and urine albumin excretion levels after treatment with vildagliptin with Vildagliptin producing a slightly higher decrease but there was no correlation with changes in A1c levels. The overall incidence of adverse experiences was low and generally mild in both groups.

Conclusions: In a group of Asian Indian patients with diabetic nephropathy due to T2DM undergoing haemodialysis in whom glycaemia was not controlled adequately, addition of gliptins helped to achieve glycaemic control to a similar extent as insulin glargine but with a marginal weight advantage.
\end{abstract}

Keywords: Comparative effectiveness, Diabetic nephropathy, Dipeptidyl peptidase-4 (DPP-4) inhibitors, Gliptins, Haemodialysis, Type 2 diabetes mellitus

\section{INTRODUCTION}

The prevalence of chronic kidney disease (CKD) is increasing worldwide with the prevalence rate higher in developing countries. ${ }^{1,2}$ Diabetic nephropathy (DN) has emerged as the common underlying cause of end-stage renal disease (ESRD) requiring dialysis in most countries. ${ }^{3}$ The progression of diabetes to advanced stages of CKD is associated with the progression of multiple other micro and macrovascular complications of diabetes. Although a number of randomized clinical trials have shown that control of hyperglycaemia, blood pressure and blockade of the renin-angiotensin-aldosterone system can slow the progression of diabetic kidney disease, good glycaemic control has emerged as the cornerstone in patients with both diabetes and CKD. ${ }^{4}$ Hence numerous drugs with different mechanisms of action and different pharmacologic profiles are being used but the evidence concerning their efficacy and safety for treating hyperglycaemia in patients with DN is sparse and the 
therapeutic options for patients with T2DM and CKD are therefore limited. ${ }^{5-7}$

Gliptins or dipeptidyl peptidase-4 (DPP-4) inhibitors have revolutionized the management of $\mathrm{T} 2 \mathrm{DM}$ and all the currently available DPP-4 inhibitors can be used not only in patients with CKD but also in patients with ESRD on dialysis. $^{5}$

The glucose-lowering effect of DPP-4 inhibitors in diabetic patients with or without CKD have been shown to be similar, but they were all based on randomized clinical trials. There are relatively few studies carried outside of clinical trials that explicitly evaluated differences in the efficacy between the various gliptins in a clinical practice setup despite the widespread prescription of these drugs among dialysis patients. ${ }^{8-11}$

The primary objective of the present study was to therefore assess the comparative effectiveness of addition of gliptins commercially available in India such as Sitagliptin or Vildagliptin to their existing therapy on glycaemic control in patients with T2DM undergoing haemodialysis as part of their routine care in a rural tertiary care setting.

\section{METHODS}

\section{Study design}

This study was a retrospective study of case records undertaken at the Dialysis Unit of our institution between March 2016 and March 2018. Studies were performed at no extra cost to the participants, and patients were not compensated. The study protocol and data extraction form were approved by the Institutional Ethics Committee.

\section{Patients}

The study patients were Asian Indian type 2 diabetes patients with DN and ESRD undergoing maintenance haemodialysis as part of routine care for their renal problems. The diagnosis of Diabetic nephropathy was made according to previously published criteria. ${ }^{12}$ The electronic records of patients who signed the consent form and who were prescribed gliptins (Sitagliptin or Vildagliptin) for control of T2DM in addition to the regular drugs and undergoing haemodialysis for their renal problems as part of routine care at our haemodialysis centre were reviewed.

Patients who were prescribed gliptins formed the study group, whereas those who were prescribed insulin glargine served as the reference group. The inclusion criteria were as broad as possible in order to maximize generalization and to reflect the "real-world" conditions. The data were extracted using a standardized data extraction form. Demographic data, along with serum creatinine, glycated haemoglobin (A1C), cholesterol, and urinary albumin creatinine ratio, and blood pressure were extracted using the standardized data extraction form.

\section{Medications}

Patients who were prescribed sitagliptin (25mg) or vildagliptin $(50 \mathrm{mg})$ used a single dose of the medication, after breakfast. Patients in the comparator group selfinjected insulin glargine once daily, and the dose was titrated against fasting plasma glucose (FPG). Patients continued their regular medications, such as antihypertensives, and lipid-lowering agents, during the study period. While the doses of other oral hypoglycaemic agents remained the same during the study period, doses of statins or blood pressure-lowering drugs were adjusted based on the patient's need.

\section{Outcome measures}

The visit when gliptin or insulin glargine were initiated was considered as baseline, and the final outcome was 24 weeks later. The primary effectiveness variable was the change in A1C level from baseline. The secondary effectiveness variables were the change from baseline in serum creatinine levels, urine albumin creatinine excretion ratio and body weight.

\section{Safety}

Safety data were collected by noting the adverse experiences recorded in the case report forms. The intensity as well as the relationship to study drug, if any, was also recorded. Symptomatic hypoglycaemia was defined as a blood glucose measurement of $<3.4 \mathrm{mmol} / \mathrm{L}$ $(<60 \mathrm{mg} / \mathrm{dL})$ and/or symptoms such as excessive hunger, sweating, shaking, palpitation, or confusion. Severe hypoglycaemia was defined as a hypoglycaemic episode in which the patient required assistance from another person or had a blood glucose measurement of $<2.8 \mathrm{mmol} / \mathrm{L}(<50 \mathrm{mg} / \mathrm{dL})$. The patients were specifically asked about symptoms of acute pancreatitis such as abdominal pain, nausea, and vomiting during each visit to the hospital. The levels of serum amylase and lipase were monitored wherever relevant.

\section{Statistical analysis}

Data are presented as mean $\pm \mathrm{SD}$ values. All data were subjected to Analysis of variance (ANOVA) and individual comparisons were made using Tukey HSD Post-hoc Test. Standard descriptive analysis, independentsamples $\mathrm{t}$ test, and nonparametric tests were used where appropriate.

\section{RESULTS}

\section{Patient characteristics}

Three hundred and forty nine diabetic patients with nephropathy and undergoing long-term haemodialysis were included in this study. The results of patient demographic and clinical parameters are listed in Table 1. Baseline demographic, clinical, and biochemical 
characteristics, as well as concomitant background therapies, were balanced between the treatment groups and did not differ significantly between the groups.

\section{Effectiveness on glycaemic control}

At the time when the patients were started on gliptins, all had uncontrolled diabetes (defined as HbAlc >6.5 (48mmol/mol) and above). However, if based on the more commonly used $\mathrm{HbA} 1 \mathrm{c}$ target of $7 \%(53 \mathrm{mmol} / \mathrm{mol})$ and above, $93.8 \%$ of the patients had uncontrolled diabetes. Baseline mean A1c, levels were comparable among the treatment groups (Table 2). There was a slight decrease in A1c values in all the three treatment groups, with the highest reduction occurring in the Vildagliptin group $(6.1 \%)$. However, this reduction reached levels of statistical significance in the gliptin treated groups only after 24 weeks of treatment.

Table 1: Baseline characteristics of the study groups.

\begin{tabular}{|llll|}
\hline Characteristic & $\begin{array}{l}\text { Sitagliptin group } \\
(\mathbf{n = 1 6 7 )}\end{array}$ & $\begin{array}{l}\text { Vildagliptin group } \\
(\mathbf{n = 1 2 4})\end{array}$ & $\begin{array}{l}\text { Insulin glargine group } \\
\mathbf{( n = 5 8 )}\end{array}$ \\
\hline Age (years) & $53.2 \pm 4.9$ & $56.4 \pm 1.8$ & $53.8 \pm 11.1$ \\
\hline Sex (M/F) (n) & $83 / 84$ & $73 / 51$ & $37 / 21$ \\
\hline Waist Circumference (cm) & $84.2 \pm 17.0$ & $83.7 \pm 16.0$ & $90.2 \pm 9.4$ \\
\hline Duration of diabetes (years) & $11.8 \pm 9.3$ & $12.2 \pm 6.8$ & $10.9 \pm 8.4$ \\
\hline Duration of dialysis (months) & $34 \pm 6$ & $35 \pm 5$ & $37 \pm 2$ \\
\hline BMI $\left(\mathrm{kg} / \mathrm{m}^{2}\right)$ & $23.2 \pm 4.4$ & $21.8 \pm 4.3$ & $22.9 \pm 3.2$ \\
\hline SBP $(\mathrm{mm} \mathrm{Hg})$ & $158.2 \pm 36.3$ & $160.8 \pm 17.1$ & $166.7 \pm 12.8$ \\
\hline DBP $(\mathrm{mm} \mathrm{Hg})$ & $97.6 \pm 12.0$ & $96.5 \pm 14.2$ & $98.2 \pm 11.7$ \\
\hline Cholesterol (mmol/L) & $6.9 \pm 1.5$ & $7.2 \pm 2.1$ & $7.4 \pm 2.8$ \\
\hline Hb $(\mathrm{g} \%)$ & $8.9 \pm 2.1$ & $8.8 \pm 3.2$ & $8.4 \pm 2.1$ \\
\hline
\end{tabular}

Table 2: Effectiveness of gliptin therapies on efficacy variables.

\begin{tabular}{|llll|}
\hline Characteristic & Sitagliptin & Vildagliptin & Insulin \\
\hline Hb A1c $(\%)$ & & & \\
\hline Control & $10.6 \pm 2.7$ & $10.3 \pm 2.4$ & $10.4 \pm 2.4$ \\
\hline 12 weeks & $10.2 \pm 0.9$ & $09.8 \pm 2.3$ & $10.1 \pm 1.9$ \\
\hline 24 weeks & $09.9 \pm 0.6^{*}$ & $09.6 \pm 1.1^{*}$ & $09.8 \pm 2.7$ \\
\hline Serum Creatinine (mmol/L) & & & \\
\hline Control & $881 \pm 209$ & $873 \pm 214$ & $896 \pm 268$ \\
\hline 12 weeks & $851 \pm 306$ & $841 \pm 426$ & $889 \pm 254$ \\
\hline 24 weeks & $831 \pm 312$ & $821 \pm 386$ & $902 \pm 264$ \\
\hline uAlb:Cr ratio $(\mathrm{mg} / \mathrm{mmol})$ & & & \\
\hline Control & $1410 \pm 272$ & $1549 \pm 236$ & $1129 \pm 389$ \\
\hline 12 weeks & $1376 \pm 392$ & $1431 \pm 432$ & $1028 \pm 296$ \\
\hline 24 weeks & $1352 \pm 364$ & $1286 \pm 764$ & \\
\hline Body weight $(\mathrm{kg})$ & & & $56.1 \pm 10.7$ \\
\hline Control & $56.8 \pm 13.6$ & $53.9 \pm 8.4$ & $56.4 \pm 10.8$ \\
\hline 12 weeks & $55.3 \pm 13.1$ & $51.2 \pm 8.5$ & $57.2 \pm 8.4$ \\
\hline 24 weeks & $55.2 \pm 12.6$ & $51.0 \pm 4.3$ & \\
\hline
\end{tabular}

\section{Effectiveness on kidney function}

There was an insignificant decrease in the serum creatinine levels after 12 and 24 weeks of treatment with gliptins with Vildagliptin producing a slightly higher decrease, suggesting an amelioration of renal function, as compared to an increase albeit a small one in the control group. Twenty four weeks of gliptin treatment reduced urinary albumin excretion as shown by a decrease in the urine albumin creatinine ratio especially in patients with high urinary albumin creatinine ratio at baseline (Table 2). It is noteworthy that these changes in albuminuria were observed despite comparable levels of HbAlc. However, there was no significant correlation between the urine albumin creatinine ratio and change in A1c levels $(\mathrm{R}=0.04$, $\mathrm{p}=0.59$ ). The reduction in serum creatinine levels coupled with reduction in urinary albumin excretion suggests that gliptin may be beneficial in patients with diabetic nephropathy undergoing haemodialysis. 


\section{Effectiveness on body weight}

After 24 weeks of therapy with additional gliptin, only small differences in body weight were observed (1.6 and $1.9 \mathrm{~kg}$ ), which did not reach the level of statistical significance (Table 2). Addition of insulin glargine led to a small weight gain of $0.9 \mathrm{~kg}$.

\section{Safety}

A total of eleven patients had mild hypoglycaemic episodes (two in the Sitagliptin group, three in the Vildagliptin group and six in the insulin group), but none was a severe episode. Sixteen patients complained of cough, rhinorrhoea, and fatigue in the gliptin group (11 in Sitagliptin and 5 in Vildagliptin group). None of the patients receiving gliptins presented with either symptoms or signs of acute pancreatitis.

\section{DISCUSSION}

With the progressively complex group of therapies offered for the management of T2DM, it is obligatory to examine the relative clinical effectiveness of diverse drug classes in the real-world setting. Although the newer agents have exhibited blood glucose-lowering efficacy in clinical trials, their effectiveness in the real-world setting is unknown. There is a well-documented gap between "best practices" established in randomized trials to improve outcomes and the care delivered in almost all primary care settings for diabetes and other chronic illnesses since the published data are based on randomized clinical trials in which patients were selected on rigorous inclusion/exclusion criteria. ${ }^{13}$ One avenue to help close this gap between research and practice is to conduct and report more "practical clinical trials or pragmatic trials" in real-world settings. Our results reflect the actual clinical practice in a large rural tertiary hospital in India.

Chen and Hsieh while studying the real-world effectiveness of sitagliptin as add-on therapy in patients with type 2 diabetes mellitus found a meaningful clinical improvement in glycaemic control after commencement of sitagliptin treatment. ${ }^{9}$ Similarly, an improvement in glycaemic control was observed in clinical trials in type 2 diabetic patients undergoing haemodialysis. ${ }^{14-17}$ In the present study, sitagliptin and vildagliptin at daily doses of 25 and 50mg per day demonstrated a comparable degree of glucose-lowering effects. This data supplements data from clinical trials and support the addition of gliptins for patients with renal impairment and undergoing haemodialysis with potential advantages of oral administration, weight neutral effect and a low risk of hypoglycaemia. Nevertheless, tangible evidences are necessary for mitigating the extra cost associated with gliptin therapy before these results can be universalised.

Preclinical observations and clinical studies suggest that DPP-4 inhibitors might also be a promising strategy for the treatment of diabetic kidney disease because of the improvement in albuminuria and possible nephronprotective effect independent of glucose lowering action. ${ }^{18}$ In this study we observed a fall albeit small one in the excretion of albumin. These promising results must be interpreted with caution and the clinical impact of this observation on renal function and its potential to influence the course of diabetic nephropathy need to be confirmed in studies involving more number of patients.

To author's knowledge, this study is the first to compare the real-world clinical effectiveness of Sitagliptin with Vildagliptin as add-ons on glycaemic control in patients undergoing HD. Our results reflect the actual clinical practice in a rural tertiary referral hospital and supplement data from randomized controlled trials. The clinical impact of DPP-4 inhibitors on renal function and their potential to influence the course of diabetic nephropathy deserve attention. Our study is limited by its relatively small sample size, and the short period of follow-up.

\section{CONCLUSION}

In conclusion, this study indicated that supplementation of gliptins may aid to accomplish glycaemic control minus the threat of hypoglycaemia. Exact underlying mechanisms still need more detailed illumination. Furthermore, studies in a large number of patients are essential to establish that DPP-4 inhibitor treatment could be used as a pharmacological measure for managing hyperglycaemia during haemodialysis. It remains to be seen whether the addition of gliptins is safe in HD population and whether it is cost saving or cost-effective.

Funding: No funding sources

Conflict of interest: None declared

Ethical approval: The study was approved by the Institutional Ethics Committee

\section{REFERENCES}

1. Tahrani AA, Barnett AH, Bailey CJ. Pharmacology and therapeutic implications of current drugs for type 2 diabetes mellitus. Nat Rev Endocrinol. 2016 Oct;12(10):566-92.

2. Vijayan M, Radhakrishnan S, Abraham G, Mathew M, Sampathkumar K, Mancha NP. Diabetic kidney disease patients on hemodialysis: a retrospective survival analysis across different socioeconomic groups. Clin Kidney J. 2016 Dec;9(6):833-8.

3. Rhee CM, Leung AM, Kovesdy CP, Lynch KE, Brent GA, Kalantar-Zadeh K. Updates on the Management of Diabetes in Dialysis Patients. Seminars in dialysis. 2014;27(2):135-45.

4. Arnouts P, Bolignano D, Nistor I, Bilo H, Gnudi L, Heaf $\mathbf{J}$, et al. Glucose-lowering drugs in patients with chronic kidney disease: a narrative review on pharmacokinetic properties. Nephrol Dial Transplant. 2014 Jul;29(7):1284-300.

5. Abe M, Okada K. DPP-4 Inhibitors in Diabetic Patients with Chronic Kidney Disease and End-Stage 
Kidney Disease on Dialysis in Clinical Practice. Contrib Nephrol. 2015;185:98-115.

6. Scheen AJ. Pharmacokinetics and clinical use of incretin-based therapies in patients with chronic kidney disease and type 2 diabetes. Clin Pharmacokinet. 2015 Jan;54(1):1-21.

7. Wang W, Zhou X, Kwong JSW, Li L, Li Y, Sun X. Efficacy and safety of thiazolidinediones in diabetes patients with renal impairment: a systematic review and meta-analysis. Sci Rep. 2017 May 11;7(1):1717.

8. Mohan Dallumal R, Chua SS, Wu DB, Vethakkan SR. Sitagliptin: Is It Effective in Routine Clinical Practice? Int J Endocrinol. 2015;2015:950571.

9. Chen TY, Hsieh CJ. Real-world effectiveness of sitagliptin as add-on therapy in patients with type 2 diabetes mellitus. Postgrad Med. 2014 May;126(3):205-15.

10. Nyeland ME, Ploug UJ, Richards A, Garcia Alvarez L, Demuth D, Muthutantri A, et al. Evaluation of the effectiveness of liraglutide and sitagliptin in type 2 diabetes: a retrospective study in UK primary care. Int J Clin Pract. 2015 Mar;69(3):281-91.

11. Ghosh S, Trivedi S, Sanyal D, Modi KD, Kharb S. Teneligliptin real-world efficacy assessment of type 2 diabetes mellitus patients in India (TREAT-INDIA study). Diabetes Metab Syndr Obes. 2016 Nov 8;9:347-53.

12. Liew BS, Perry C, Boulton-Jones JM, Simpson K, Paterson K. Diabetic nephropathy: an observational study on patients attending a joint diabetes renal clinic. QJM. 1997 May;90(5):353-8.

13. Sudhakaran C, Kishore U, Anjana RM, Unnikrishnan R, Mohan V. Effectiveness of sitagliptin in Asian Indian patients with type 2 diabetes-an Indian tertiary diabetes care center experience. Diabetes Technol Ther. 2011 Jan;13(1):27-32.

14. Ito M, Abe M, Okada K, Sasaki H, Maruyama N, Tsuchida M, et al. The dipeptidyl peptidase-4 (DPP-4) inhibitor vildagliptin improves glycemic control in type 2 diabetic patients undergoing hemodialysis. Endocr J. 2011;58(11):979-87.

15. Arjona Ferreira JC, Corry D, Mogensen CE, Sloan L, $\mathrm{Xu}$ L, Golm GT, et al. Efficacy and safety of sitagliptin in patients with type 2 diabetes and ESRD receiving dialysis: a 54-week randomized trial. Am J Kidney Dis. 2013;61(4):579-87.

16. Kume S, Uzu T, Takagi C, Kondo M, Okabe T, Araki $\mathrm{S}$, et al. Efficacy and tolerability of vildagliptin in type 2 diabetic patients on hemodialysis. J Diabetes Investig. 2012;3(3):298-301.

17. Ito H, Mifune M, Matsuyama E, Furusho M, Omoto T, Shinozaki M, et al. Vildagliptin is Effective for Glycemic Control in Diabetic Patients Undergoing either Hemodialysis or Peritoneal Dialysis. Diabetes Ther. 2013;4(2):321-9.

18. Penno G, Garofolo M, Del Prato S. Dipeptidyl peptidase-4 inhibition in chronic kidney disease and potential for protection against diabetes-related renal injury. Nutr Metab Cardiovasc Dis. 2016 May;26(5):361-73.

Cite this article as: Suthakaran $\mathrm{C}$, Bhaskaran S. Comparative effectiveness of sitagliptin and vildagliptin in the management of patients with type 2 diabetes mellitus undergoing haemodialysis: an Indian rural tertiary care centre experience. Int $\mathbf{J}$ Basic Clin Pharmacol 2018;7:1268-72. 